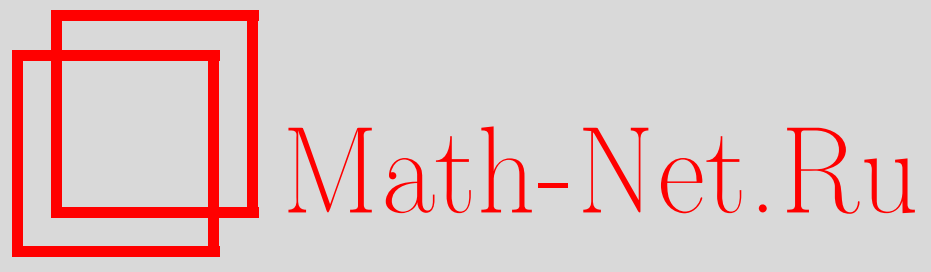

А. В. Петухов, Геометрическое описание эпиморфных подгрупп, УМН, 2010, том 65, выпуск 5, 193-194

DOI: https://doi.org/10.4213/rm9384

Использование Общероссийского математического портала Math-Net.Ru подразумевает, что вы прочитали и согласны с пользовательским соглашением http://www . mathnet.ru/rus/agreement

Параметры загрузки:

IP: 54.80 .97 .219

26 апреля 2023 г., $17: 44: 33$ 


\section{Геометрическое описание эпиморфных подгрупп}

\section{А. В. Петухов}

1. Введение. Пусть $G$ - аффинная алгебраическая группа над алгебраически замкнутым полем $\mathbb{K}$ характеристики 0 и $H$ - ее подгруппа. Тогда стабилизатор всех вектор-функций для правого действия $G$ на $\mathbb{K}[G]^{H}$ есть $\widehat{H}$, обозримая оболочка $H$ в $G$ [1]. Для всякого $G$-модуля $V$ верно, что $V^{H}=V^{\widehat{H}}$ [1]. Это показывает интерес вычислений обозримых оболочек подгрупп и, в частности, определения подгрупп $H$, для которых $\widehat{H}=G,-$ эпиморфных подгрупп [2]. Подгруппа называется обозримой, если $\widehat{H}=H$.

ОПРедЕлЕниЕ 1 . Пусть $G$ - полупростая алгебраическая группа, а $H$ - eе peдуктивная подгруппа. Тогда ортогональным централизатором к $H$ в $G$ называется подгруппа $\mathrm{Z}_{G}^{\perp}(H)$, удовлетворяющая условиям

1) группа $Z_{G}^{\perp}(H)$ связна;

2) lie $\mathrm{Z}_{G}^{\frac{1}{G}}(H)=\{z \in \operatorname{lie} G$ : для любого $h \in \operatorname{lie} H$ выполнены равенства $[z, h]=0$ и $(z, h)=0\}$, где $(\cdot, \cdot)$ - форма Картана-Киллинга полупростой алгебры Ли lie $G$.

Каждой подгруппе $H$ с алгеброй Ли $\mathfrak{h}$ группы $G$ с алгеброй Ли $\mathfrak{g}$ можно сопоставить вектор $v_{H} \in \operatorname{Hom}_{\mathbb{C}}(\mathfrak{h}, \mathfrak{g})$, соответствующий естественному вложению $H$ в $G$. Заметим, что пространство $\operatorname{Hom}_{\mathbb{C}}(\mathfrak{h}, \mathfrak{g})$ наделяется структурой $G$-модуля с помощью присоединенного действия группы Ли $G$ на $\mathfrak{g}$. Здесь и далее $\mathrm{R}_{\mathrm{u}}(H)$ - унипотентный радикал группы $H$, а $\mathrm{L}_{H}$ - произвольная подгруппа Леви группы $H$.

Теорема 1. Нередуктивная подгруппа $H$ полупростой группы $G$ обозрима в ней тогда и только тогда, когда замыкание орбиты $\mathrm{Z}_{G}^{\perp}\left(\mathrm{L}_{H}\right) v_{\mathrm{R}_{\mathrm{u}}(H)}$ содержит 0.

Tеорема 2. Подгруппа $H$ эпиморфна тогда и только тогда, когда орбита $\mathrm{Z}_{G}^{\perp}\left(\mathrm{L}_{H}\right) v_{\mathrm{R}_{\mathrm{u}}(H)}$ замкнута и $H$ не содержится в собственной редуктивной подгруппе группы $G$.

2. Доказательства теорем 1 и 2. Здесь и далее $G$ - полупростая алгебраическая группа с алгеброй Ли g.

ОПРЕдЕлЕниЕ 2. Полупростой элемент $s \in \mathfrak{g}$ называется рациональным, если оператор его присоединенного действия на $\mathfrak{g}$ обладает рациональными собственными значениями.

Пусть $s \in \mathfrak{g}$ - рациональный полупростой элемент. Пространство $\mathfrak{g}$ есть $\bigoplus_{i \in \mathbb{Q}} \mathfrak{g}_{i}$, где $\mathfrak{g}_{i}$ - собственные ad $s$-подпространства с собственными значениями $i$. Пространства $\mathfrak{p}_{s}=\bigoplus_{i \geqslant 0} \mathfrak{g}_{i}, \mathfrak{q}_{s}=\left\{p \in \mathfrak{p}_{s}:(p, s)=0\right\}, \mathfrak{n}_{s}=\bigoplus_{i>0} \mathfrak{g}_{i}$ являются алгебраическими подалгебрами Ли в $\mathfrak{g}$. Алгебра $\mathfrak{n}_{s}$ является нильпотентным радикалом $\mathfrak{p}_{s}$ и $\mathfrak{q}_{s}$. Связная алгебраическая группа $\mathrm{Q}_{s}$ с алгеброй Ли $\mathfrak{q}_{s}$ называется квазипараболической.

Теорема 3 (критерий Суханова). Подгруппа $H \subset G$ обозрима в $G$ тогда и толъко тогда, когда существует рациональный полупростой элемент $s \in \mathfrak{g}$ такой, что lie $H \subset \mathfrak{q}_{s} u$ lie $\mathrm{R}_{\mathrm{u}}(H) \subset \mathfrak{n}_{s}$.

СледСтвие 1. Подгруппа $H \subset G$ эпиморфна в $G$ тогда и толъко тогда, когда она нередуктивна и lie $H$ не вложена ни в какую подалгебру $\mathfrak{q}_{s}$ ни для какого ненулевого рачионального полупростого элемента $s \in \mathfrak{g}$.

ПредлОЖЕНИЕ 1. Пусть $H^{\prime} \subset H \subset G$ - цепочка вложенных алгебраических групп, причем $H$ и $H^{\prime}$ редуктивны. Тогда $\mathrm{Z}_{G}^{\perp}(H) \subset \mathrm{Z}_{G}^{\perp}\left(H^{\prime}\right)$. 
ЗАмечание 1 . Пусть подгруппа $H^{\prime}$ вложена в подгруппу $H$ группы $G, \mathrm{R}_{\mathrm{u}}\left(H^{\prime}\right) \subset$ $\mathrm{R}_{\mathrm{u}}(H), 0 \in \overline{\mathrm{Z}_{G}^{\perp}\left(\mathrm{L}_{H}\right) v_{\mathrm{R}_{\mathrm{u}}(H)}}$. Тогда $0 \in \overline{\mathrm{Z}_{G}^{\perp}\left(\mathrm{L}_{H^{\prime}}\right) v_{\mathrm{R}_{\mathrm{u}}\left(H^{\prime}\right)}}$, где $\mathrm{L}_{H^{\prime}} \subset \mathrm{L}_{H}-$ подгруппы Леви групп $H^{\prime}$ и $H$ соответственно.

ДокАЗАТЕЛЬСтво ПРЕДЛожЕния 1. Верно, что $\mathrm{Z}_{G}^{\perp}\left(\mathrm{L}_{H}\right) \subset \mathrm{Z}_{G}^{\perp}\left(\mathrm{L}_{H^{\prime}}\right)$, откуда искомое утверждение и следует.

ПредлоЖение 2. Пусть $s \in \mathfrak{g}$ - рационалъный полупростой элемент. Тогда $0 \in$ $\overline{\mathrm{Z}_{G}^{\perp}\left(\mathrm{L}_{\mathrm{Q}_{s}}\right) v_{\mathrm{R}_{\mathrm{u}}\left(\mathrm{Q}_{s}\right)}}$.

ДокАзАтельство. Пусть $\bar{s}: \mathbb{K}^{*} \rightarrow G$ - однопараметрическая подгруппа, соответствующая $s$. Тогда $\lim _{t \rightarrow 0} \bar{s}(t) v_{\mathrm{R}_{\mathrm{u}}\left(\mathrm{Q}_{s}\right)}=0$.

Следствие 2. Если подгруппа $H$ группы $G$ обозрима в $G$, mо $0 \in \overline{\mathrm{Z}_{G}^{\perp}\left(\mathrm{L}_{H}\right) v_{\mathrm{R}_{\mathrm{u}}(H)}}$.

ПРедЛОЖениЕ 3. Если для подгруппъ $H$ группъ $G$ верно, что $0 \in \overline{\mathrm{Z}_{G}^{\perp}\left(\mathrm{L}_{H}\right) v_{\mathrm{R}_{\mathrm{u}}(H)}}$, то она обозрима в $G$.

ДокАЗАТЕЛьСтво. Пусть $s \in \mathfrak{g}$ - рациональный полупростой элемент, а $V$ - представление группы $G$. Тогда $V=\bigoplus_{i \in \mathbb{Q}} V_{i}^{s}$, где $V_{i}^{s}$ - это $s$-собственные подпространства с собственными значениями $i$.

По критерию Гильберта-Мамфорда существует ненулевой рациональный полупростой элемент $s \in \operatorname{lie} \mathrm{Z}_{G}\left(\mathrm{~L}_{H}\right)$ такой, что $v_{\mathrm{R}_{\mathrm{u}}(H)} \in \bigoplus_{i>0} \operatorname{Hom}_{\mathbb{K}}\left(\operatorname{lie} \mathrm{R}_{\mathrm{u}} H, \mathfrak{g}\right)_{i}^{s}$, значит, lie $\mathrm{R}_{\mathrm{u}} H \subset \mathfrak{n}_{s}$ и lie $\mathrm{R}_{\mathrm{u}} H \perp s$. Следовательно, lie $\mathrm{H} \subset \mathfrak{q}_{s}$.

Следствие 2 и предложение 3 вместе составляют теорему 1.

ПредлОЖенИЕ 4. Пусть $\widehat{H}$ нередуктивна. Тогда орбита $\mathrm{Z}_{G}^{\perp}\left(\mathrm{L}_{H}\right) v_{\mathrm{R}_{\mathrm{u}} H}$ незамкнута.

ДокАЗАТЕЛьСтво. По критерию Суханова существует рациональный полупростой элемент $s \in \mathfrak{g}$ такой, что lie $\widehat{H} \subset \mathfrak{q}_{s}, \quad \operatorname{lie} \mathrm{R}_{\mathrm{u}} \widehat{H} \subset \mathfrak{n}_{s}$. Тогда $\lim _{t \rightarrow 0} v_{\mathrm{R}_{\mathrm{u}} H} \in$ $\operatorname{Hom}\left(\operatorname{lie} H, \operatorname{lie} \mathrm{L}_{\widehat{H}}\right)$. Если этот элемент лежит в $\mathrm{Z}_{G}^{\perp}\left(\mathrm{L}_{H}\right) v_{H}$, то $H^{0} \subset \mathrm{L}_{\widehat{H}}$. Заметим, что $\operatorname{dim} \mathrm{L}_{\widehat{H}}<\operatorname{dim} \widehat{H}$.

ПредЛожениЕ 5. Пусть нередуктивная группа $H$ эпиморфна в $G$. Тогда орбита $Z_{G}^{\perp}\left(\mathrm{L}_{H}\right) v_{\mathrm{R}_{\mathrm{u}} H}$ замкнута.

ДокАзАтельство. Предположим противное. По критерию Гильберта-Мамфорда существует рациональный полупростой элемент $s \in \mathrm{Z}_{G}^{\perp}\left(\mathrm{L}_{H}\right)$ такой, что $v_{\mathrm{R}_{u} H} \in$ $\bigoplus_{i \geqslant 0} \operatorname{Hom}_{\mathbb{K}}\left(\operatorname{lie} \mathrm{R}_{\mathrm{u}} H, \mathfrak{g}\right)_{i}^{s}$. Следовательно, lie $\mathrm{R}_{\mathrm{u}} H \subset \mathfrak{p}_{s}$. Пусть $\mathrm{P}_{s}-$ алгебраическая подгруппа Ли в $G$ с алгеброй Ли $\mathfrak{p}_{s}$. Тогда $\mathrm{R}_{\mathrm{u}} H \subset \mathrm{P}_{s}$. Легко видеть, что $\mathrm{P}_{s} / \mathrm{Q}_{s} \cong \mathbb{K}^{*}$, и, следовательно, $\mathrm{R}_{\mathrm{u}} H \subset \mathrm{Q}_{s}$, откуда lie $H \subset \mathfrak{q}_{s}$.

Предложения 4 и 5 составляют доказательство теоремы 2.

\section{Список литературы}

[1] F. D. Grosshans, Algebraic homogeneous spaces and invariant theory, Lecture Notes in Math., 1673, Springer-Verlag, Berlin, 1997. [2] F. Bien, A. Borel, C. R. Acad. Sci. Paris. Ser. I Math., 315 (1992), 1341-1346.

\section{А. В. Петухов (А. V. Petukhov)}

Московский государственный университет им. М. В. Ломоносова;

Jacobs University, Bremen, Germany

E-mail: a.petukhov@jacobs-university.de
Представлено А. В. Михалёвым Принято редколлегией 01.08.2010 